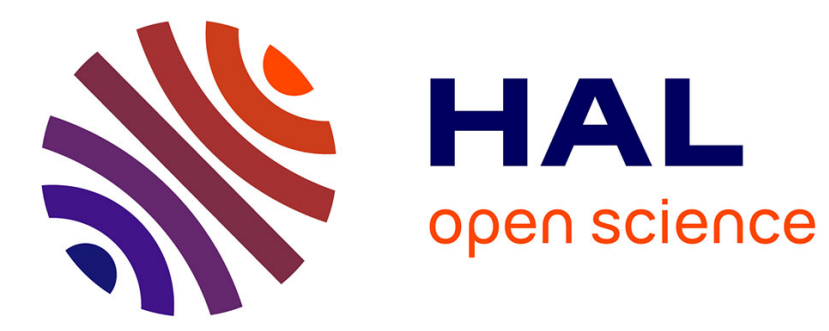

\title{
Amour et gloire dans Les Aventures de Télémaque
}

François Trémolières

\section{To cite this version:}

François Trémolières. Amour et gloire dans Les Aventures de Télémaque. Littératures classiques, 2009, $\mathrm{N}^{\circ} 70$ (3), pp.297. 10.3917/licla.070.0297 . hal-02304406

\section{HAL Id: hal-02304406 \\ https://hal.univ-rennes2.fr/hal-02304406}

Submitted on 4 Oct 2019

HAL is a multi-disciplinary open access archive for the deposit and dissemination of scientific research documents, whether they are published or not. The documents may come from teaching and research institutions in France or abroad, or from public or private research centers.
L'archive ouverte pluridisciplinaire HAL, est destinée au dépôt et à la diffusion de documents scientifiques de niveau recherche, publiés ou non, émanant des établissements d'enseignement et de recherche français ou étrangers, des laboratoires publics ou privés. 


\section{AMOUR ET GLOIRE DANS LES AVENTURES DE TÉLÉMAQUE}

\section{François Trémolières}

Armand Colin | «ittératures classiques »

2009/3 Nº 70 | pages 297 à 313

ISSN 0992-5279

ISBN 9782908728613

Article disponible en ligne à l'adresse :

https://www.cairn.inforevue-litteratures-classiques1-2009-3-page-297.htm

Distribution électronique Cairn.info pour Armand Colin.

(C) Armand Colin. Tous droits réservés pour tous pays.

La reproduction ou représentation de cet article, notamment par photocopie, n'est autorisée que dans les limites des conditions générales d'utilisation du site ou, le cas échéant, des conditions générales de la licence souscrite par votre établissement. Toute autre reproduction ou représentation, en tout ou partie, sous quelque forme et de quelque manière que ce soit, est interdite sauf accord préalable et écrit de l'éditeur, en dehors des cas prévus par la législation en vigueur en France. Il est précisé que son stockage dans une base de données est également interdit. 


\section{François Trémolières}

\section{Amour et gloire dans Les Aventures de Télémaque}

Il n’est pas facile d’être le héros du Télémaque ${ }^{1}$. Non seulement parce que l'auteur multiplie les épreuves et les «aventures » pour son personnage - mais encore parce que l'héroïsme lui-même est suspect, en cette fin de siècle fatiguée des guerres et des fastes ludoviciens ${ }^{2}$ : laminé par les critiques moralistes et jansénistes ${ }^{3}$, tout aussi profondément atteint par la spiritualité du pur amour ${ }^{4}$. Aussi voit-on Télémaque, qui vient pourtant de s’illustrer au combat, paraître «embarrassé et incertain » - au point, dit le texte, d'en rougir - lorsque l'assemblée des rois ligués contre Adraste lui adresse des louanges :

1 Nous citerons le Télémaque dans l'édition établie par J.-L. Goré, officiellement choisie pour l'agrégation de lettres 2010 (Les Aventures de Télémaque, Paris, Garnier, 1987, rééd. 2009) mais en indiquant aussi les références dans celle procurée par J. Le Brun pour la collection de la Pléiade (dans Fénelon, Euvres, Paris, Gallimard, 1983-1997, t. II). Elles seront notées respectivement par les lettres OP et G, les pages étant indiquées en chiffres arabes. Nous avons modernisé l’orthographe mais nous ne signalons pas les variations entre les deux éditions, notamment de ponctuation.

2 On se souviendra que Fénelon a rédigé, anonymement, la fameuse Lettre à Louis XIV [décembre 1693 ?] : voir la préface de Fr.-X. Cuche, « Un prophète à la Cour », à l'édition qu'il en a donnée (Rezé, Séquences, 1994). Sur ce climat fin de siècle, voir J. Cornette, Le Roi de guerre. Essai sur la souveraineté de la France du Grand Siècle, Paris, Payot, 1993, «Épilogue ». La date de rédaction des Aventures de Télémaque, publiées sans l'autorisation ni le nom de l'auteur en 1699, n’est pas connue avec précision : J. Le Brun propose 16921693.

Voir les thèses désormais classiques de Paul Bénichou sur « la démolition du héros », Morales du Grand Siècle, Paris, Gallimard, 1948.

4 La présente étude reprend une partie du dernier chapitre de notre thèse, à paraître chez Champion sous le titre Fénelon et le sublime. Littérature, anthropologie, spiritualité. Voir aussi «Le sublime dans le Télémaque », dans Fénelon. Mystique et politique (16991999), Paris, Champion, 2004, p. 255-274.

Littératures Classiques, 70, 2010 
Ce n’est pas que je les aime, surtout quand elles sont données par de si bons juges de la vertu ; mais c'est que je crains de les aimer trop ; elles corrompent les hommes ; elles les remplissent d'eux-mêmes ; elles les rendent vains et présomptueux. Il faut les mériter et les fuir. [...] Les bonnes louanges seront celles que vous me donnerez en mon absence, si je suis assez heureux pour en mériter. Si vous me croyez véritablement bon, vous devez croire aussi que je veux être modeste et craindre la vanité. $^{5}$

Mais le Télémaque n'est pas un livre comme les autres: il est écrit ad usum Delphini $^{6}$ - pour le duc de Bourgogne, petit-fils du roi, destiné à la couronne, dont Fénelon est alors le précepteur... La relation entre le prince et son éducateur se trouve ainsi reproduite dans celle entre Mentor et Télémaque, dont les commentateurs ont souligné l'orientation "pédagogique ${ }^{7}$ ». Certains traits du personnage de Télémaque peuvent s'expliquer par la projection d'un caractère réel en particulier son emportement, furor qu'aurait tendance à magnifier l'épopée, mais que Fénelon-Mentor prend soin de brider ${ }^{8}$. Or la question de la « réputation » n’est pas de celles que l'on peut négliger, là où la «gloire » du meneur d'hommes apparaît souvent comme une condition de sa réussite. Louis XIV notait dans ses Mémoires pour 1667 :

Les rois, qui sont nés pour posséder tout et commander tout, ne doivent jamais être honteux de s'assujettir à la renommée : c'est un bien qu'il faut désirer sans cesse avec plus d'avidité, et qui seul, en effet, est plus capable que tous les autres de servir le succès de nos desseins. ${ }^{9}$

5 G 506 / OP 278. «Les meilleures louanges ressemblent aux fausses », celles des flatteurs, que met en question le supplice des « mauvais rois » aux Enfers avec la mention des " poètes » (l’ode politique) et des « orateurs » (le panégyrique) : G 456-457 / OP 242-243.

6 Sur la réception du Télémaque et les changements de perception induits par la publication, voir V. Kapp, Télémaque de Fénelon. La signification d'une œuvre littéraire à la fin du siècle classique, Tübingen / Paris, G. Narr / J.-M. Place, 1982 ; et à sa suite les travaux d'Emmanuel Bury, notamment « Situation du Télémaque. Du projet pédagogique à la fortune littéraire », dans Fénelon. Mystique et politique (1699-1999), op. cit., p. 533-547.

Notamment Jacques Le Brun : OP 1254. Sur le caractère ad hominem du Télémaque, voir Fr.-X. Cuche, Télémaque entre père et mer, $2^{\mathrm{e}}$ éd., Paris, Champion, 1995, p. 32.

8 L’adjectif furieux a un sens élevé dans le vocabulaire héroïque - complètement retourné par Fénelon : voir par exemple G 411 / OP 211. Les appels à la «patience » dans l'éducation de Télémaque, l’oxymore de la «patience héroïque », ont aussi cette dimension pédagogique, ad hominem, de redresser un caractère (voir parmi les opuscules pédagogiques de Fénelon, «Le Fantasque », Euvres, éd. cit., t. I, p. 258) - et l’on sait, notamment par Saint-Simon, que Fénelon y a réussi (Mémoires, éd. Y. Coirault, Paris, Gallimard, " Bibliothèque de la Pléiade », 1983-1993, t. IV, p. 409-428).

9 Cité d'après Y. Durand : L'Ordre du monde. Idéal politique et valeurs sociales en France du XVI $I^{e}$ au XVIII siècle, Paris, Sedes, 2001, p. 67. Voir aussi J.-P. Labatut, Louis $X I V$, Roi de gloire, Paris, Imprimerie nationale, 1984 ; J.-P. Néraudau, L'Olympe du RoiSoleil. Mythologie et idéologie royale au Grand Siècle, Paris, Les Belles-Lettres, 1986. 
S'en prendre à la « gloire », c'est donc viser « la fine pointe de tout l'édifice de la monarchie française »: telle est la thèse de Marc Fumaroli, d'un Fénelon entreprenant de transformer la royauté en changeant « le cœur » du prince ${ }^{10}$.

\section{Le cycle guerrier}

Il faut les mériter et les fuir: nous sommes aux antipodes ici de l'éthique aristotélicienne, qui veut que le magnanime se reconnaisse digne de louange ${ }^{11}$. Plus tôt dans le livre, Télémaque, alors que la guerre contre les Dauniens n’a pas encore révélé sa " valeur ${ }^{12}$ » (virtu), entend les louanges ou plutôt les actions de grâce de soldats $^{13}$, qu'il a donné ordre de soigner et de réconforter :

Il sentait ce plaisir si doux et si pur que les dieux ont attaché à la seule vertu et que les méchants, faute de l'avoir éprouvé, ne peuvent ni concevoir ni croire ; mais il ne s'abandonnait point à ce plaisir [...] ; il n'oubliait point sa hauteur naturelle et son indifférence pour les hommes; il avait une honte secrète d'être né si dur, et de paraître si humain. Il renvoyait à la sage Minerve toute la gloire qu'on lui donnait, et qu'il ne croyait pas mériter. ${ }^{14}$

À son retour des combats, il apprendra de Mentor la leçon :

Vous avez fait de grandes fautes ; mais elles vous ont servi à vous connaître et à vous défier de vous-même. ${ }^{15}$

Les grandes actions enflent le cœur et inspirent une présomption dangereuse ; les fautes font rentrer l'homme en lui-même et lui rendent la sagesse. ${ }^{16}$

10 Voir M. Fumaroli, Le Poète et le Roi. Jean de la Fontaine en son siècle, Paris, de Fallois, 1997, notamment p. 434-435.

11 Ou de «l'honneur» (timè) dans l'Éthique à Nicomaque: voir R.-A. Gauthier, Magnanimité. L'idéal de la grandeur dans la philosophie païenne et dans la théologie chrétienne, Paris, Vrin, 1951, en particulier p. 78-86.

12 Comme la «réputation », le terme est réinvesti par Fénelon : voir G 133, 329, 496 / OP 13, 153, 271.

13 À leur insu, sans quoi elles seraient suspectes : voir G 432-433 / OP 227-228.

14 G 433 / OP 228. Voir l'émouvante lettre de Fénelon au P. Martineau après la mort du duc de Bourgogne, décrivant un élève selon son cœur : «Je ne l'ai jamais vu aimer les louanges. Il les laissait tomber d'abord, et si on lui en parlait, il disait simplement qu'il connaissait trop ses défauts pour mériter d'être loué. » (14 novembre 1712, Correspondance de Fénelon, éd. J. Orcibal, t. XVI, Genève, Droz, 1999, p. 101).

15 G 520 / OP 288.

16 G 525 / OP 292. Cette sagesse est discernement : l'homme de bien reconnaît l'action divine dans ce qu'il fait de bon. Mentor / Minerve est ainsi une métaphore de la grâce. 
Aussi Mentor se montre-t-il heureux de retrouver Télémaque «si désabusé des victoires et des conquêtes, dans un âge où il était si naturel qu'il fût enivré de la gloire qu'il avait acquise ». Cette ascèse doit gagner tout le corps social, sans quoi celui-ci risquerait la ruine. Précisément la vertu du chef (la tête de ce corps) a pour effet d'éviter la corruption des mœurs, c'est-à-dire la dissolution du lien social : par l'exemple (c'est le thème platonicien du « roi philosophe ${ }^{17}$ ») et surtout par l'instauration puis le respect des lois, l'exercice de la justice pour son peuple et de l'arbitrage pour les autres nations ; d’où la supériorité constamment affirmée dans le Télémaque $^{18}$ du législateur sur le conquérant (et l'importance d'une bonne paix après une guerre). Télémaque l'avoue : «ce que vous avez fait ici [Mentor à Salente] est infiniment plus grand que les victoires que nous venons de remporter $^{19}$ ». La gloire au combat a toujours quelque chose de hasardeux : c'est même le théâtre par excellence de l'intervention de la Providence, ou des dieux ${ }^{20}$. Ainsi la signification profonde du courage héroïque est-elle pour Fénelon de consentir à remettre sa vie entre les mains de Dieu : le roi doit être prêt à mourir au combat. Cette mort ne doit pas être crainte. Ainsi Télémaque pleure Hippias, dans une formule paradoxale (qui peut rappeler les pages d'Augustin dans les Confessions sur la mort de Monique ${ }^{21}$ ): «Si je ne t'aimais, j'envierais ton bonheur $^{22}$.» Elle ne sanctionne pas nécessairement les mauvais (la logique du combat n'est pas celle de l'ordalie) - le cas extrême étant au contraire le

17 G 524 / OP 291.

18 Notamment dans la figure de Minos, «le plus sage et le meilleur de tous les rois » (G 195 / OP 58), «père » de la législation de la Crète et juge aux Enfers. Voir aussi la réponse de Télémaque prétendant justement au trône crétois (G 207-210 / OP 67-69).

19 G 525 / OP 292. Cf. G 332 / OP 156 (« il faudrait des dieux pour redresser les hommes »).

20 Rivalités de Minerve avec Mars (G 495, 569 / OP 271, 324), avec Vénus (G 225-226, 275-276 / OP 80-81, 114-115), avec Neptune... Voir G. Ferreyrolles, « La Providence dans le Télémaque ", dans Fénelon. Mystique et politique (1699-1999), p. 197 : le « consentement» de Jupiter (G 276 / OP 115) à la vengeance de Vénus, « limité et calculé », est «l'analogue exact » de la réponse de Dieu à Satan sur le sort de Job : "Va, tout ce qu'il a est en ton pouvoir ; mais je te défends de porter la main sur lui » (Job, I, 12).

21 Confessions, IX, 12, trad. Arnauld d'Andilly, Paris, Gallimard, « Folio », p. 323 sq.

22 G 435 / OP 230 ; ce «bonheur » d'être mort au combat fait la douleur de ceux qui survivent (Phalante, Phérécyde, Télémaque). 
"dévouement ${ }^{23}$ ", qui au prix d'une vie donne la victoire: sacrifice qui est précisément attendu du soldat ${ }^{24}$.

Sans doute « ce que les hommes appellent grandeur, gloire, profonde politique » ne paraît du haut de l'Olympe «que misère et faiblesse ${ }^{25}$ ». Mais il n'y en a pas moins dans le Télémaque un cycle épique, avec les livres XIII à XV, qui autour du séjour aux Enfers (livre XIV) mettent en scène une sorte de transfiguration du héros. Le livre XIII commence par une caricature de combat : le duel quasi fratricide de Télémaque avec Hippias : laissé à lui-même, en l'absence de Mentor, Télémaque ne sait où jeter sa gourme, son ardeur " frénétique» et furieus $\mathrm{e}^{26}$; il garde de sa victoire imméritée une "honte secrète " - « une douleur que rien ne pouvait consoler $^{27}$ ». La guerre contre les Dauniens vient l'effacer : «Télémaque, qui était abattu et inconsolable, oublie sa douleur », « un feu divin étincelle dans les yeux du jeune guerrier ${ }^{28} » \ldots$ Cette première phase du combat s'ouvre sur la mort héroïque d'Hippias (signal d'ailleurs du « réveil » de Télémaque) et se conclut sur un orage qui permet à Adraste d'échapper à la défaite et prépare aux alliés de nouvelles épreuves. Surtout, ce suspens providentiel est mis à profit par Télémaque pour aller chercher son père dans les Enfers (livre XIV). Au livre XV, à la reprise de la bataille, "Minerve avait mis dans ses yeux un feu divin, et sur son visage une majesté fière qui promettait déjà la victoire », la déesse « avait mis je ne sais quoi de terrible dans sa voix » : véritable apothéose du héros, qui triomphe d'Adraste au combat $^{29}$.

La guerre est parfois nécessaire pour convaincre les mauvais «qu'il y a des dieux » et les obliger à les craindre. Le bon ne combat que malgré lui, sait qu'il ne

23 Sur la devotio antique, voir J. Le Brun, Le Pouvoir d'abdiquer. Essai sur la déchéance volontaire, Paris, Gallimard, 2009, chap. 2. Voir aussi l'opuscule spirituel de Fénelon «Sur le pur amour», Euvres, éd. cit., t. I, p. 668 («Cette idée du parfait désintéressement [jusqu'à la mort volontaire] régnait dans la politique de tous les anciens législateurs ») ; et son commentaire par J. Le Brun : Le Pur amour, de Platon à Lacan, Paris, Éditions du Seuil, 2002, p. 23-47.

24 Accepter la mort, qui est l'attitude requise au combat, signifie simplement que la vie a moins de prix que la "vertu », c'est-à-dire doit être subordonnée à l'ordre divin ; mais puisqu'il est impossible à l'homme d'identifier la volonté divine dans le cours du monde, la plus grande sagesse est l'abandon à Dieu (que le Télémaque prend bien soin de distinguer de toute passivité, au sens de découragement). Une politique chrétienne n’a donc pas de plus profonde maxime que ce «but unique et essentiel » que Mentor fixe au prince : « ne vouloir jamais l'autorité et la grandeur pour soi [...] se sacrifier, dans les peines infinies du gouvernement, pour rendre les hommes heureux » (G 553 / OP 312).

25 G 274 / OP 113-114. Voir aussi G 470 / OP 253.

26 G 409-411 / OP 210-211. Même comparaison avec un cheval fougueux pour Bocchoris : G 152 / OP 27.

$27 \quad$ G 416 / OP 215.

28 G 423 / OP 220 ; G 426 / OP 223. C’est là que figure la description du bouclier, « don précieux de Minerve », dont Télémaque ne sait pas encore qu'elle est Mentor.

29 G 487 / OP 265 ; G 495 / OP 271. 
se doit pas à lui-même la victoire. C'est délégitimer le « roi de guerre ». Avant la bataille décisive, Télémaque invoque Jupiter en ces termes :

C'est à regret que nous combattons; nous voudrions épargner le sang des hommes ; nous ne haïssons point cet ennemi même [Adraste], quoiqu'il soit cruel, perfide et sacrilège. Voyez et décidez entre lui et nous : s'il faut mourir, nos vies sont dans vos mains; s'il faut délivrer l'Hespérie et abattre le tyran, ce sera votre puissance et la sagesse de Minerve, votre fille, qui nous donnera la victoire ; la gloire vous en sera due. ${ }^{30}$

Certes le chef victorieux apparaît « au-dessus de l'humanité ; mais tout cela n'est que merveilleux, tout cela ne fait que nous étonner ${ }^{31} »$. C'est autre chose qui au livre XVI rend Télémaque apte à régner :

Il est humain, il est bon, il est ami fidèle et tendre [...]; il s'est défait de sa hauteur, de son indifférence et de sa fierté. ${ }^{32}$

Si donc la guerre doit être un théâtre de mort (une manifestation de la dépendance absolue de l'être créé, incapable de se donner la vie), si le sort des combats (la victoire ou la défaite) comme le sort des individus (vivre ou mourir) ne dépend pas des hommes - sinon sans doute à proportion de leur « courage ", c'est-à-dire leur mépris de la mort -, ce qui dépend d'eux en revanche c'est que la guerre soit «juste» dans ses motifs ${ }^{33}$ : ici nous retrouvons la responsabilité propre des puissants, qui n'est pas seulement de veiller à ne pas pervertir le mépris de la mort en mépris de la vie, le courage en "fureur », le consentement à mourir en désir de tuer, mais aussi, mais surtout, de n’engager la guerre que par nécessité : «Le sang d'un peuple ne doit être versé que pour sauver ce peuple dans les besoins extrêmes ${ }^{34}$. »

Dans le long argument, au début du livre XV, où Télémaque de retour des Enfers se révèle à la fois un grand orateur ${ }^{35}$ et un chef, Fénelon réfute tout ce qui pourrait,

30 G 488 / OP 266.

31 G 507 / OP 279. On reconnaît là les termes mêmes qui caractérisent le sublime dans la préface de Boileau à sa traduction de Longin : " cet extraordinaire et ce merveilleux qui frappe dans le discours »; termes qui dès les Dialogues sur l'éloquence (écrits dans les années 1670) suscitaient des réserves chez Fénelon.

32 G 507 / OP 279.

33 La mauvaise guerre ramène toujours à l’hybris du roi. La guerre juste se déduit nécessairement d'une situation où elle apparaît, pour les peuples dont le roi a la charge, comme le moindre mal.

34 Réflexions de Télémaque au livre XIII : G 430 / OP 226.

35 On reproche parfois au Télémaque son côté bavard: mais c'est oublier qu'ici la parole est action, et qu'une bonne part de l'éducation du héros consiste à l'encourager à " persuader» et à préférer le débat, l'échange, l'alliance au combat. La leçon n'est pas si « chimérique » à en juger par ce qu'en feront les Lumières: de «miroir du prince », le 
non plus dans le motif mais dans la manière même de combattre, faire qu'une cause juste permette le triomphe de l'injustice : chaque fois c'est encore le mépris de la mort qui est le critère. Lorsque Démophante permettrait d'entrer par ruse dans une ville adverse, au mépris de la parole donnée, Télémaque s'insurge ${ }^{36}$; lorsque Acanthe est soupçonné sans preuve de vouloir l'empoisonner et qu'on se propose, par précaution, d'exécuter le suspect, même indignation ${ }^{37}$. Ce modèle de roi vertueux, qui préfère la mort à la fraude, au parjure ou à l’injustice, enseigne aux alliés en quoi leur propre intérêt ${ }^{38}$ est de refuser de tels moyens. Ainsi de la trahison, car « dès que nous l'aurons autorisée par notre exemple, nous mériterons qu'elle se tourne contre nous ${ }^{39} »$.

Si l'amour de la vertu, et la crainte des dieux ne vous touchent plus, au moins soyez touchés de votre réputation et de votre intérêt. [...] Quelle sûreté pourrez-vous donner quand vous voudrez être sincères et qu'il vous importera de persuader à vos voisins votre sincérité ? [...] Vous n’aurez plus de ressource pour faire croire ce que vous promettrez. ${ }^{40}$

Ce qui vaut pour les nations vaut $a$ fortiori pour les individus qui les forment :

Voici, ajouta Télémaque, un intérêt encore plus pressant qui doit vous frapper, s’il vous reste quelque sentiment de probité et quelque prévoyance sur vos intérêts : c'est qu'une conduite si trompeuse attaque par le dedans toute votre ligue et va la ruiner [...] Comment [...] pouvez-vous vous confier les uns aux autres, si une fois vous rompez l'unique lien de la société et de la confiance, qui est la bonne foi ? Après que vous aurez posé pour maxime qu'on peut violer les règles de la probité et de la fidélité pour un plus grand intérêt, qui d'entre vous pourra se fier à un autre, quand cet autre pourra trouver un grand avantage à lui manquer de parole et à le tromper ? [...] Que devient une ligue de tant de peuples, lorsqu'ils sont convenus entre eux par une délibération commune, qu'il est permis de surprendre son voisin et de violer la foi donnée ? [...] Ô rois sages et magnanimes [...] si vous aviez une fois rompu la barrière de l'honneur et de la bonne foi, cette perte est irréparable. ${ }^{41}$

Télémaque est devenu pour ses lecteurs du XVIII ${ }^{\mathrm{e}}$ siècle le miroir du pouvoir des idées - de leur propre pouvoir; et Fénelon, pour Robespierre, rien moins que le «précepteur de l'humanité ».

36 G 479 / OP 259 : « ne vaut-il pas mieux périr que de vaincre par de tels moyens ? ».

37 G 483-485 / OP 262-263.

38 Comme la « réputation », cet intérêt illustre la logique de ce que Fénelon appelle dans les écrits spirituels « amour mélangé », par opposition à l'amour pur ou « sans aucun mélange du motif de l'intérêt propre » (Explication des maximes des saints, Euvres, éd. cit., t. I, p. 1011).

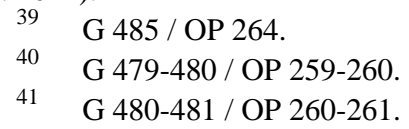


Ainsi la guerre juste est-elle même préférable, malgré tout ce que l'on sait des horreurs qu'elle engendre ${ }^{42}$, à son évitement par la ruse ${ }^{43}$. La civilisation (la vie en société) apparaît comme une conquête permanente sur l'esprit de discorde, fruit de l'orgueil et de l'amour-propre ${ }^{44}$ : comme l'écrit excellemment Philippe Sellier, « la rêverie édénique réussit à fleurir sur les armes elles-mêmes ${ }^{45}$ ». L'art de gouverner suppose une sorte de refondation - ou pour mieux dire de fondation perpétuelle ${ }^{46}$ du lien social, dont le roi est l'artisan, la «mesure fixe » : à lui de se faire l'étalon ou le parangon de la justice, car les méchants

ne sont point des hommes incapables de faire le bien ; ils le font indifféremment, de même que le mal, quand il peut servir à leur ambition. (...) À proprement parler, ils ne sont pas capables de la vertu, quoiqu'ils paraissent la pratiquer ; mais ils sont capables d'ajouter à tous leurs autres vices le plus horrible des vices, qui est l'hypocrisie $^{47}$

- et l'hypocrisie ou la dissimulation, c'est-à-dire le contraire de la «bonne foi », sont pour Fénelon à la fois une menace pour la société et l'ordre des mœurs, et l'antinomie de toute politique chrétienne.

42 Voir la véhémente harangue de Mentor au livre IX : G 305-306 / OP 135- 136. Fénelon insiste ailleurs sur le caractère providentiel des épreuves de la guerre, manifestation du « Dieu terrible » : G 390 et 570 / OP 197 et 325.

43 Voir le discours de Télémaque au livre $\mathrm{XV}$, qui aboutit à faire prendre conscience que le combat avec Adraste est inévitable : G 478-480 / OP 258-261.

44 Voir en particulier l'épisode de la Bétique : G 267-269 / OP 110-111.

45 Voir supra Ph. Sellier, «La résistance à l'épopée : Les Aventures de Télémaque ». Il s’agit d'une allusion à la représentation, sur le bouclier de Télémaque, des origines de la civilisation : naissance d'Athènes, enfance des arts, peinture de «l'âge d'or » (G 423-425 / OP 221-222)... On sait qu'elle s'est substituée à une première version (G 439-442 / OP 14261429), plus longue, représentant l'histoire d'Édipe et des siens. Cette violence du mythe fondateur, Fénelon la réinvente dans l'histoire d’Idoménée, «meurtre du fils » qui n’est pas sans écho non plus avec sa méditation de la Passion : voir J. Le Brun, «Fénelon. Un fils est tué » [1989] dans La Jouissance et le trouble, Genève, Droz, 2006, p. 513-531 ; « Idoménée et le meurtre du fils. Le trompe-l'œil de l'utopie », dans Fénelon. Philosophie et spiritualité, Genève, Droz, 1996, p. 77-93.

46 Nous disons refondation pour bien marquer que la politique de Fénelon ne légitime aucunement une révolution. Au contraire, et de la manière la plus claire, elle fait obligation aux sujets de fidélité à leur souverain - même dans le cas de Pygmalion, tyran sanguinaire et débauché (G 161 / OP 33). Si advient une révolution, c'est, au même titre que la guerre, comme un châtiment divin, dont le souverain est le véritable coupable (G 349 / OP 168). Après la transformation de Mentor, Minerve déclare à Télémaque : « La crainte est nécessaire quand l'amour manque ; mais il la faut toujours employer à regret » (G 570 / OP 325).

47 G 368-369 / OP 182. Sur la «mesure fixe », voir la leçon platonicienne du livre XVIII : G 553/ OP 312. 
La mort au combat n'est donc que la version « héroïque ${ }^{48}$ », spectaculaire, ostentatoire, du sacrifice exigé du souverain. Tout au plus, les grands sont-ils plus que d'autres tentés par l'hybris : car confrontés au risque de la mort, comment ne céderaient-ils pas, proprement médusés ${ }^{49}$ par leur force (l'usage licite de la violence), à la fureur de tuer ? Ce pouvoir de vie et de mort sur autrui, l'excès de la puissance, avec ce qu'elle entraîne de louanges et de flatterie, font que la grandeur épique est particulièrement exposée au mal, qu'on pourrait définir comme emprise du néant et de la mort. Ce serait même une dernière ruse de l'amour-propre que de rendre plus attrayante la mort au combat que la modération dans la conduite de l'État ${ }^{5}$. Néanmoins les rois, comme les autres hommes, ne peuvent attendre que d'une ascèse toute personnelle - précisément ce qu'on appelle amour de la vertu ce dépassement de «l'intérêt propre » qui purifie de tout orgueil le désir de mourir. Car la mort est désirable, mais d'un désir qu'il n'est pas permis d'assouvir.

\section{Le cycle amoureux}

Telle est l'étrange vérité qu'insinue l'incipit du Télémaque :

Calypso ne pouvait se consoler du départ d’Ulysse. Dans sa douleur, elle se trouvait malheureuse d'être immortelle.

Ce chagrin de la déesse, à qui la consolation de la mort est interdite, elle croit pouvoir s'en défaire en aimant Télémaque, mais cet amour lui étant aussi refusé, la plainte se répète, lorsque Mentor, au livre VI, force le fils d'Ulysse à quitter son île : ainsi se termine un premier cycle d'épreuves pour le héros (les six premiers livres), que nous pourrions appeler cycle de Calypso ${ }^{51}$. Plusieurs fois dans ces pages

48 De même que l'abdication en est la version tragique : en vertu de la conception dynastique, elle ne peut qu'être voulue par le roi, un acte dû à son libre consentement, sans quoi il ne s'agit que d'usurpation ou de révolution. Voir les Mémoires politiques sur la succession d'Espagne (Euvres, éd. cit., t. II, p. 1013 sq.) et le commentaire qu'en donne J. Le Brun (Le Pouvoir d'abdiquer, chap. 8).

49 Ainsi « la pâle Mort conduite par une Furie infernale, dont la tête est hérissée de serpents » (G 422 / OP 219), accompagne Adraste au combat. Voir O. Leplatre, « Le sacrifice de Méduse. Autour de la mort de Bocchoris (livre II) ", L’École des lettres, LXXXVI / 4, 15 nov. 1994, p. 39-58.

50 Voir au début du livre IX le discours de Mentor à un Télémaque « plein d’une noble ardeur pour les combats » : la prudence, la sagesse et la modération d’Ulysse ont mieux servi la victoire que le « courage bouillant et farouche » d'Achille (G 293-294 / OP 127) : « une si belle passion pour la gloire » doit, comme toutes les passions, être dominée et purifiée. Voir aussi la fausse alternative « de périr ou de vaincre » (G 303 / OP 134).

51 Fr. Raviez a récemment insisté sur l'importance de ces livres dans l'éducation du duc de Bourgogne : « Mourir à l'amour au vif de l'amour. Télémaque au péril des flots ", Nord', $n^{\circ} 45$, avril 2005, p. 51-62. 
«Télémaque aspire à la mort ${ }^{52}$ ». Mais c'est Mentor le premier (ce sont quasiment ses premiers mots) qui affirme que «le naufrage et la mort sont moins affreux que les plaisirs qui attaquent la vertu ${ }^{53}$ ». Télémaque fait rapidement la preuve de sa qualité d'âme en préférant à la servitude le sacrifice de sa vie : "cette condition [d'esclave] me parut plus dure que la mort ${ }^{54} »$. Il cède pourtant à un véritable désespoir :

Il ne me restait plus aucune espérance [...] la douleur me pressant, j'oubliais un jour mon troupeau et je m'étendis sur l'herbe auprès d'une caverne où j'attendais la mort, ne pouvant plus supporter mes peines. ${ }^{55}$

Il faudra l'intervention vraiment providentielle d'une voix sortie de la caverne (et dont Télémaque rendra grâce à Minerve) pour le tirer de cette mortelle torpeur ${ }^{56}$.

52 Note de J. Le Brun, OP 1328. Nous préférons l'expression de « désir de mort » (id., «Préface » à l’éd. Folio du Télémaque, Paris, Gallimard, 1995, p. 23) à celle de « pulsion de mort » utilisée par Fr.-X. Cuche (Télémaque entre père et mer, p. 79-80) et que nous serions tenté pour notre part de réserver à ce que nous venons de nommer « fureur de tuer ». Le seul cas, nous semble-t-il, où Fénelon risque l'expression de "plaisir de mourir » concerne la mort des méchants, encore est-ce dans la variante abandonnée de l'ekphrasis du bouclier de Télémaque (G 442 / OP 1429).

53 G 124 / OP 6. L’insistance de Mentor à combattre le « venin mortel » de la volupté (G 186 / OP 52) et sa difficulté à convaincre le héros de fuir les plaisirs pourraient signaler un enjeu plus caché : éviter tout amalgame de la fameuse « douceur » fénelonienne avec quelque forme d'hédonisme, ou de "plaisir sensible ». Enjeu particulièrement net dans le récit de l'apparition onirique de Vénus - qui n'est pas sans rappeler... la description des états mystiques par Bossuet («mes sens étaient liés et suspendus », G 179 / OP 47) - et dans le soin mis à produire des contre-figures féminines : Amphitrite (G 191-192 / OP 56) opposée à Vénus (A. Blanc, «Fonction de la référence mythologique dans le Télémaque », XVII siècle, $\mathrm{n}^{\circ} 125,1979, \mathrm{p}$. 373-388), ou Antiope à Eucharis (G 530-531 / OP 296).

54 G 130 / OP 11.

55 G 142-143 / OP 20. Après cette crise se trouve placé l'épisode de Termosiris (deuxième père de substitution), qui l'encourage à transformer sa condition d'esclave en celle de berger, "plus douce que toute [la] gloire» des rois (G 147 / OP 23) ; puis un épisode hérö̈que : sa victoire sur le lion, tel un nouvel Hercule (G 148 / OP 24-25).

56 La question de l'espérance (vertu dont les théologiens ont redouté l'absence dans la spiritualité du pur amour, voir infra, n. 62) mériterait pour le Télémaque une étude particulière. Tout le livre a ceci d'eschatologique qu'il raconte une quête dont le dénouement (le retour à Ithaque et la reconnaissance d'Ulysse) se trouve hors de lui. Dès le début Télémaque est partagé entre l'inquiétude (le mouvement des «aventures") et le découragement : il se dit « lassé de vivre en suspens et toujours dans l'incertitude » (G 127 / OP 9). Lorsqu'au livre II la captivité en Égypte lui fait penser qu' «il n’y a plus d’Ithaque pour nous ", il déclare à son compagnon : " Mourons, mon cher Mentor ; nulle autre pensée ne nous est plus permise, mourons, puisque les dieux n’ont aucune pitié de nous »; déjà Mentor ne peut y répondre que par une intervention de style prophétique : « Indigne fils du sage Ulysse $[\ldots]$ vous vous laissez vaincre à votre malheur! Sachez que vous reverrez un 
Mais Télémaque cède assez vite à une «profonde tristesse » lorsque, fait prisonnier à Tyr, il envie le sort des naufragés. Cette tristesse n’est cependant pas le désespoir qu'au même moment Bocchoris dans sa « fureur » livre en spectacle : elle est plutôt consentement à son destin. Ainsi, au livre V («la mort se présenta à nous $\left.{ }^{57} »\right)$, c'est la répétition de l'épreuve de la tempête, sur laquelle s'ouvrait le livre ${ }^{58}$ :

J'embrasse Mentor, et je lui dis : «Voici la mort, il faut la recevoir avec courage. Les dieux ne nous ont délivrés de tant de périls que pour nous faire périr aujourd'hui. Mourons, Mentor, mourons. C'est une consolation pour moi de mourir avec vous. Il serait inutile de disputer notre vie contre la tempête. » Mentor me répondit : « Le vrai courage trouve toujours quelque ressource. Ce n’est pas assez d'être prêt à recevoir tranquillement la mort, il faut, sans la craindre, faire tous ses efforts pour la repousser. [...] Croyez-vous, Télémaque, que votre vie soit abandonnée aux vents et aux flots? Croyez-vous qu'ils puissent vous faire périr sans l'ordre des dieux ? Non non; les dieux décident de tout. C'est donc les dieux, et non pas la mer, qu'il faut craindre. ${ }^{59}$

La signification de l'épreuve est si peu ici le refus de la mort que la narration met en scène aussitôt après (livre VI) le refus de l'immortalité en échange de l'amour :

« Quoi donc ! » disait-il à Mentor, les larmes aux yeux, « vous ne comptez pour rien l'immortalité qui m'est offerte par la déesse ? - Je compte pour rien, répondit Mentor, tout ce qui est contre la vertu, et contre les ordres des dieux [...] Hé ! que feriez-vous d'une vie immortelle sans liberté, sans vertu et sans gloire ? Cette vie serait encore plus malheureuse en ce qu'elle ne pourrait finir. » 60

jour l'île d'Ithaque et Pénélope. Vous reverrez même [...] l’invincible Ulysse » (G 137-138 / OP 16-17). La voix qui sort de la caverne assure de même : " Tu reverras Ithaque, et ta gloire montera jusqu'aux astres [...] sache que tu ne seras grand qu'autant que tu seras modéré et courageux pour vaincre tes passions » (G 143 / OP 20-21).

57 G 219 / OP 76.

58 Il s'agit en effet de la même tempête, celle qui jette les héros sur l'île de Calypso, mais cette fois prise dans le récit de Télémaque, au terme de la longue parenthèse où il a raconté à la déesse ses aventures antérieures : une première boucle est bouclée, la fin du cycle approche. La deuxième boucle sera le retour du dépit amoureux de Calypso. Les deux épisodes sont liés, peut-on dire, par métonymie : le récit par Télémaque de la tempête et ce que S. Guellouz appelle le « récit premier » («Structure narrative du Télémaque », dans Je ne sais quoi de pur et de sublime...Télémaque, Orléans, Paradigme, 1994, p. 53-76) avec la description du trouble de son cœur «comme la mer, qui est le jouet de tous les vents contraires » (G 230 / OP 84).

59 G 220-221 / OP 76-77.

60 G 229 / OP 83. Mentor pourrait offrir ici la figure d'un dieu qui consent à mourir véritable tour de force dans le cadre de la fable : la mort d'Hercule, au livre XII (G 391-392 / OP 198), est celle d'un demi-dieu, dont la « double nature » manque un peu de finesse. 
Ce refus entraîne la malédiction de Calypso, c'est-à-dire la mise à nu d'un désir de mort (mort de l'autre) par une véritable perversion (ou inversion) du désir de mourir :

Non, non, que jamais tu ne revoies ta patrie, cette pauvre et misérable Ithaque, que tu n'as pas eu honte de préférer à l’immortalité ! Ou plutôt que tu périsses, en la voyant de loin, au milieu de la mer ; et que ton corps, devenu le jouet des flots, soit rejeté, sans espérance de sépulture, sur le sable de ce rivage ! [...] Ô si j’étais libre de me donner la mort pour finir mes douleurs! Télémaque, il faut que tu meures, puisque je ne puis mourir $!^{61}$

Est-il permis de remarquer, dans l'attitude de Calypso consentant finalement au départ de Télémaque (ce qui est le rendre à sa condition de mortel), une certaine noblesse, qui faisait la beauté douloureuse de l'incipit ? Son état ressemble assez alors au désespoir du "pur amour » : aimer sans retour, et pour l'éternité, un dieu absent s'il nous condamnait à l'enfer ${ }^{62}$. Télémaque - et c'est la dernière étape de ce cycle - devra encore pour sa part se désabuser de l'amour qu'il porte à Eucharis. Incapable de ne pas céder au trouble amoureux, il implore Mentor comme son « vrai père » : « Délivrez-moi de tant de maux. Délivrez-moi de moi-même. Donnez-moi la mort. » Mentor rappelle le héros à lui-même («Fils du sage Ulysse ») et surtout « lui apprend à se supporter lui-même » : « Les dieux vous ont conduit comme par la main jusqu'au bord de l'abîme, pour vous en montrer toute la profondeur, sans vous y laisser tomber ${ }^{63}$.» Télémaque, qui semble apaisé, demande alors à faire ses adieux à la nymphe :

Au moins souffrez que je lui dise : « Ô nymphe, les dieux cruels, les dieux jaloux de mon bonheur me contraignent de partir. Mais ils m’empêcheront plutôt de vivre

\footnotetext{
61 G 232-234 / OP 85-86.

62 Qu'il faille aimer Dieu quand bien même il nous livrerait à la damnation, telle est la «supposition impossible » dont les auteurs spirituels du XVII ${ }^{\mathrm{e}}$ siècle font le critère de la pureté d'intention du mystique. On peut rapprocher la douleur de Calypso de celle des damnés du livre XIV, qui implorent la mort comme une délivrance, quand aux Enfers « on ne peut mourir » (G 458 / OP 244). La parenté du pur amour avec l'amour des poèmes et des romans est déjà relevée par Cervantès (Don Quichotte, I, 31) : « Il faut que tu saches [dit Don Quichotte à Sancho Pança] qu'en notre style de chevalier c'est un grand honneur à une dame d'avoir plusieurs chevaliers errants qui la servent, sans que leurs pensées s'étendent à plus qu'à la servir pour son seul mérite, sans espérer autre récompense de tous leurs bons désirs, si ce n'est qu'elle se contente de les accepter pour ses chevaliers. - J'ai ouï prêcher, dit Sancho, que c'est de cette sorte d'amour qu'il faut aimer Notre-Seigneur, pour lui seul, sans y être porté d'aucune espérance de gloire, ou de crainte de peine » (trad. C. Oudin, Paris, Gallimard, «Folio », 1976, p. 373).

63 G 239 / OP 90. Cette image de l’abandon est fréquente dans les écrits spirituels.
} 
que de me souvenir à jamais de vous. » Ô mon père, ou laissez-moi cette dernière consolation, qui est si juste, ou arrachez-moi la vie en ce moment. ${ }^{64}$

Mentor réplique avec «pitié » : «Vous croyez être tranquille, et vous demandez la mort ! [...] Pourquoi voulez-vous mourir ${ }^{65}$ ? » Il lui faut employer, pour en finir, la manière forte - une forme de violence qui ressemble elle-même à la mort ${ }^{66}$ :

Aussitôt le sage Mentor, poussant Télémaque, qui était assis sur le bord du rocher, le précipite dans la mer, et s’y jette avec lui. Télémaque, surpris de cette violente chute, but l'onde amère et devint le jouet des flots. Mais, revenant à lui et voyant Mentor qui lui tendait la main pour l'aider à nager, il ne songea plus qu'à s'éloigner de l’île fatale.

Télémaque en tire lui-même la leçon :

Je ne crains plus ni mer, ni vents, ni tempêtes [ni la mort, faut-il comprendre] ; je ne crains plus que mes passions. L'amour est lui seul plus à craindre que tous les naufrages. ${ }^{67}$

C'est alors (livre VII) que se place l'épisode du «chant divin » de Mentor l'une des rares occurrences du sublime dans le Télémaque (et la seule à ne pas désigner le séjour des élus) :

La voix de Mentor n’avait aucune douceur efféminée ; mais elle était flexible, forte, et elle passionnait jusqu'aux moindres choses. Il chanta d'abord les louanges de Jupiter, père et roi des dieux et des hommes, qui, d'un signe de sa tête, ébranle l'univers. Puis il représenta Minerve qui sort de sa tête, c'est-à-dire la sagesse, que ce dieu forme au-dedans de lui-même et qui sort de lui pour instruire les hommes dociles. [...] Ensuite il chanta le malheur du jeune Narcisse, qui, devenant follement amoureux de sa propre beauté, qu'il regardait sans cesse au bord d'une fontaine, se consuma lui-même de douleur et fut changé en une fleur qui porte son nom. Enfin il

64 G 240 / OP 91. On remarquera que par amour pour Eucharis Télémaque est prêt à mourir, alors que par défaut d'amour pour Calypso il refusait l'immortalité : c’est une première étape du consentement à sa condition.

65 G 240-241 / OP 92.

66 Cf. Montaigne, Essais (II, 3, allusion finale aux Tusculanes) : « Mais on désire aussi quelquefois la mort pour l'espérance d'un plus grand bien. Je désire, dit saint Paul, être dissout pour être avec Jésus-Christ [cupio dissolvi (Philip., I, 23)] ; et : Qui me déprendra de ces liens ? Cleombrotus Ambraciota, ayant lu le Phédon de Platon, entra en si grand appétit de la vie advenir que, sans autre occasion, il alla se précipiter dans la mer ». Voir M. Screech, Montaigne et la mélancolie [1983], Paris, P.U.F., 1992, p. 59 sq..

67 G 244-245 / OP 94. Ajoutons que ce que nous avons appelé « cycle guerrier » dans le roman commence par un rappel des méfaits de l'amour, et de la grandeur de l'amitié : le récit, au début du livre XII, de la mort d'Hercule, causée par sa passion pour Iole et la jalousie de Déjanire (G 389 / OP 196). 
chanta aussi la funeste mort du bel Adonis, qu’un sanglier déchira, et que Vénus, passionnée pour lui, ne put ranimer en faisant au ciel des plaintes amères. ${ }^{68}$

Refus d'assimiler le verbe à la douceur « efféminée », c'est-à-dire à ce qui serait condamnable dans le plaisir (refus, aussi, d'assimiler le pathétique à la mièvrerie) ; variété, force, et ce caractère propre à la poésie, de " passionner » ce qu'elle peint : l'essentiel d'une « poétique » fénelonienne est ici contenu ${ }^{69}$. Quant aux thèmes du chant, ils sont également remarquables: la louange des dieux ${ }^{70}$, la mort comme fascination de l'amour-propre (Narcisse meurt de n'aimer que soi) ou sanction des dérèglements de la passion (l’immortelle Vénus n'a pas le pouvoir de rendre la vie). Les commentateurs ont relevé ce qu'avait de déroutant ce choix, après le début « majestueux » du passage, qui laisse attendre quelque grand morceau de poésie : aussi peut-on y voir un contre-pied délibéré à l'épopée, la volonté d'en décevoir l'attente chez le lecteur, autant qu'une manière de clore le cycle pour ainsi dire lyrique des premiers livres ${ }^{71}$.

L'essentiel en tout cas est que Fénelon y impose une forme nouvelle d'éloquence, et qui lui est propre : «Tous ceux qui l'écoutèrent ne purent retenir leurs larmes, et chacun sentait je ne sais quel plaisir en pleurant ${ }^{72}$ ». Ce goût d'un pathétique particulier, à la fois douloureux et attendrissant, trouvait déjà à s'exprimer, au livre VI, dans l'évocation des larmes de Vénus, « la douleur répandant sur son visage de nouvelles grâces ${ }^{73}$ "... Mais n'est-ce pas elle qui nous était donnée à entendre dès le début, véritable diapason du style fénelonien ? Cette note vibrante de l'incipit, le lamento de Calypso, se propage dans tout le livre tendu d'emblée entre le suspens d'un début in medias res et la certitude d'une fin

68 G 261-262 / OP 105. Voir « Le sublime dans le Télémaque », art. cit. supra n. 4.

69 Cette promotion d'une «peinture » passionnée se trouve par exemple dans la Lettre à l’Académie (Euvres, éd. cit., t. II, p. 1165-1167) et déjà théorisée dans les Dialogues sur l'éloquence (ibid., t. I, p. 34-35).

70 Ou plutôt du premier d'entre eux (le plus proche du Dieu unique) et de sa « fille » la Sagesse biblique (Proverbes, VIII, 30).

71 Voir J. Le Brun, OP 1362, n. 7 et 8. Adonis en particulier, viril, héroïque, mais dont la mort tient de la parodie de combat, peut apparaître comme la figure d'une contre-épopée ; quant à Vénus, son échec achève de réduire à rien les promesses d’immortalité de Calypso...

72 G 262 / OP 105 (c'est nous qui soulignons). Sur ce thème, voir A. Lanavère, « Les larmes de Télémaque », dans Je ne sais quoi de pur et de sublime... Télémaque, p. 173-184.

73 G 226 / OP 81 (elle s'adresse à son fils Cupidon). Une première fois, au livre V, elle était apparue « avec douleur ; ses beaux yeux étaient baignés de larmes » (devant Neptune, G 219 / OP 76), formule reprise au début du livre VIII, lorsqu'elle revient implorer Jupiter (G 275 / OP 114). On lit aussi que « l'affliction augmente la beauté d’Eucharis » (G 238 / OP 90). Pour de possibles références picturales, voir "Sentiment sur différents tableaux », Euvres, éd. cit., t. I, p. 267-268. Cette figure de Vénus peut apparaître comme un relais dans la sensibilité et l'iconographie entre la Madeleine repentante, tant représentée au XVII ${ }^{\mathrm{e}}$ siècle et selon Bremond «la sainte du pur amour », et le type créé par Greuze au siècle suivant, si apprécié par Diderot, de la Jeune fille qui pleure un oiseau mort... 
connue d'avance $^{74}$ : temps incertain de la vie, temps arrêté de la mort, ou de l'éternité. Nous la retrouvons dans la «complaisance attendrie ${ }^{75}$ » des nombreuses scènes de deuil : vieillards pleurant leurs enfants morts, jeunes héros rendant un dernier hommage à leurs compagnons - note singulière, différente aussi bien de la couleur stridente, furieuse, de l'épopée, que de la basse sombre, funèbre, de l'oraison $^{76}$. Ainsi de ce passage émouvant (et cruel) du livre XVI, où Nestor, inconsolable de la mort de son fils Pisistrate ${ }^{77}$, ne trouve quelque réconfort que «par le plaisir de raconter ce qu'il avait souffert » à Diomède, et de partager avec lui sa souffrance $^{78}$. Mais c'est surtout en deux endroits décisifs ${ }^{79}$, la descente aux Enfers (livre XIV) et la rencontre avec l'inconnu (livre XVIII), que cette note devient insistante, paroxystique. Il s’agit, lors du premier épisode, de décrire l'effet sur le héros du discours de son aïeul Arcésius :

Ces paroles entraient jusqu'au fond du cœur de Télémaque [...]. Ces sages paroles étaient comme une flamme subtile, qui pénétrait dans les entrailles du jeune Télémaque : il se sentait ému et embrasé ; je ne sais quoi de divin semblait fondre son cœur au-dedans de lui. Ce qu’il portait dans la partie la plus intime de lui-même le consumait secrètement. Il ne pouvait ni le contenir, ni le supporter, ni résister à une si

74 Les Aventures de Télémaque se donnent dans les premières éditions comme une Suite du quatrième livre de l'Odyssée, comme une incise.

75 Nous reprenons l'expression à J. Le Brun (Les Aventures de Télémaque, Préface de l'éd. Folio, p. 13-14). Voir G 435, 493, 494 / OP 230, 269, 270 (morts d'Hippias, Pisistrate, Amphimaque); et Ph. Sellier, «Fleurs qui se fanent, fleurs tranchées. Essai sur l'imaginaire des Aventures de Télémaque » [1998], Essais sur l’imaginaire classique, op. cit., p. 329-344.

76 On est loin en effet de ce qu’A. Michel (« La grandeur et l’humilité. La Bible dans l'esthétique littéraire française », dans Le Grand Siècle et la Bible, Paris, Beauchesne, 1989) appelle le «sublime noir » de l'oraison funèbre, ce « sublime de la mort » chez Bossuet, plus en rapport sans doute avec «l'obsession baroque de la mort» étudiée par l'historien M. Vovelle et qui chez Fénelon se trouverait plutôt dans des passages où, selon l'expression de Jacques Le Brun, il sait «théâtraliser l'horreur » : combats épiques, mort des méchants (Bocchoris, Astarbé) ; passages qui relèvent donc selon nous, plus de l'esthétique baroque que du sublime au sens moderne, d'un « terrible » burkien ou même kantien.

77 « Il n’y a que moi qui ne puis mourir », dit-il en pleurant son fils (G 493 / OP 270).

78 G 514 / OP 284. Diomède, valeureux capitaine à la recherche d'un royaume, poursuivi par la vengeance divine, est une figure errante, qui rappelle à Télémaque son propre père, et annonce celle du pseudo-Cléomène. Cette vertu de compassion est illustrée ailleurs dans le livre ; voir par exemple la rencontre avec Cléomène : «L'exemple d'un homme encore plus malheureux que lui adoucissait la peine de Télémaque » (G 564 / OP 320).

79 Il faudrait en ajouter un troisième, à la fin du livre XVII, mais qui concerne Idoménée : sa tentative de faire de Télémaque son fils par substitution, puis l'échec de celleci, peuvent être lus comme une sorte de répétition de l'initiation de Télémaque lui-même, ou d'initiation parallèle. 
violente impression. C'était un sentiment vif et délicieux, qui était mêlé d'un tourment capable d'arracher la vie. ${ }^{80}$

Enfin, avec la rencontre du pseudo-Cléomène au dernier livre, le discours de Mentor se trouve accompagné d'une sorte d'intrigue parallèle ${ }^{81}$ qui loin de le parasiter, comme elle en a l'apparence, est au contraire nécessaire, et doit être menée à son terme, pour que le contenu de ce discours soit « incorporé82 » : histoire d'une émotion, ressentie par Télémaque à la vue d'un inconnu arrivé par la mer, depuis son apparition sur le rivage jusqu'au départ de son navire.

Alors une impression secrète de douleur saisit le cœur de Télémaque ; il s’afflige sans savoir pourquoi ; les larmes coulent de ses yeux, et rien ne lui est si doux que de pleurer. [...] Un étonnement et un trouble secret tient ses yeux attachés vers ce vaisseau déjà parti, dont il ne voit plus que les voiles, qui blanchissent un peu dans l'onde azurée. Il n'écoute pas même Mentor qui lui parle et il est tout hors de luimême [...]. Enfin, il revient un peu de cette espèce d'enchantement, et les larmes recommencent à couler de ses yeux. ${ }^{83}$

La leçon de ces variations nous semble claire : il faut consentir à une sorte de désespérance (le mot est fort, mais nous n’en trouvons pas de meilleur), la chérir même comme ce qu'il y a de meilleur en nous, de plus intime, pour ouvrir à la vérité son chemin. C’est l'équivalent littéraire exact de la "préparation du cœur» qu’enseignaient les écrits spirituels :

un sentiment confus de notre impuissance, un désir de ce qui nous manque, un penchant à trouver au-dessus de nous ce que nous cherchons en vain au dedans de nous-mêmes, une tristesse sur le vide de notre cœur, une faim et une soif de vérité. ${ }^{84}$

On voit qu'une étude complète du lien entre la spiritualité de Fénelon et Les Aventures de Télémaque devrait s’arrêter au détail de l’organisation du récit s’interroger en particulier, comme nous venons de le suggérer, sur la place de

80 G 467 / OP 250-251 (nous soulignons). Il est possible de faire le rapprochement avec l'expérience mystique (Fr.-X. Cuche, Télémaque entre père et mer, op. cit., p. 256) - mais de notre point de vue, à la condition de considérer cette dernière comme anticipation de la mort, et non pas de la vision béatifique. Sur cette dimension, d'ailleurs classique (le « mourir de ne pas mourir » chez Thérèse d'Avila : Muero porque no muero), voir P.-L. Landsberg, Essai sur l'expérience de la mort [1936], Paris, Éditions du Seuil, « Points », 1993, p. 86 sq.

81 G 557-564 / OP 315-321.

82 Nous reprenons le mot très fort employé à propos de la lumière au livre XIV : elle « s'incorpore » aux justes « comme les aliments s'incorporent à nous » (G 461 / OP 246).

83 G 564-565 / OP 321 (c'est nous qui soulignons).

84 Fénélon, Lettres sur divers sujets concernant la religion et la métaphysique, VI, Euvres, éd. cit., t. II, p. 811. 
l'expérience des Enfers (livre XIV) dans cette économie de la narration, et la signification, à la fin du roman, des deux rencontres successives : avec Cléomène (Ulysse) puis avec Minerve (transfiguration de Mentor) ${ }^{85}$. Il ne s'agissait ici que de montrer en quoi les deux cycles narratifs que nous avons décrits obéissaient à une nécessité dans la transformation de Télémaque, c'est-à-dire dans l'éducation du prince : la prise en compte des expériences majeures de l'amour et de la gloire.

François Trémolières Université de Paris Ouest - Nanterre - La Défense

85 Voir J.-L. Goré, « Le Télémaque, périple odysséen ou voyage initiatique » [1963], et A. Blanc, "Au dernier livre du Télémaque : rencontre du père ou passage du divin ? " [1980], études reprises dans Je ne sais quoi de pur et de sublime...Télémaque, op. cit. Voir également Fr.-X. Cuche, Télémaque entre père et mer, et notre article "Les fins du Télémaque », dans Lectures de Fénelon : Les Aventures de Télémaque, dir. J. Garapon et I. Trivisani-Moreau, Rennes, Presses Universitaires de Rennes, 2009. 